\title{
Genome-wide investigation and expression profiling of polyphenol oxidase (PPO) family genes uncover likely functions in organ development and stress responses in Populus trichocarpa
}

Fang He ${ }^{*}{ }^{+}$, Yu-Jie Shi ${ }^{\dagger}$, Qian Zhao, Kuang-Ji Zhao, Xing-Lei Cui, Liang-Hua Chen, Han-Bo Yang, Fan Zhang, Jia-Xuan Mi, Jin-Liang Huang and Xue-Qin Wan*

\begin{abstract}
Background: Trees such as Populus are planted extensively for reforestation and afforestation. However, their successful establishment greatly depends upon ambient environmental conditions and their relative resistance to abiotic and biotic stresses. Polyphenol oxidase (PPO) is a ubiquitous metalloproteinase in plants, which plays crucial roles in mediating plant resistance against biotic and abiotic stresses. Although the whole genome sequence of Populus trichocarpa has long been published, little is known about the PPO genes in Populus, especially those related to drought stress, mechanical damage, and insect feeding. Additionally, there is a paucity of information regarding hormonal responses at the whole genome level.

Results: A genome-wide analysis of the poplar PPO family was performed in the present study, and 18 PtrPPO genes were identified. Bioinformatics and GRT-PCR were then used to analyze the gene structure, phylogeny, chromosomal localization, gene replication, cis-elements, and expression patterns of PtrPPOs. Sequence analysis revealed that two-thirds of the PtrPPO genes lacked intronic sequences. Phylogenetic analysis showed that all PPO genes were categorized into 11 groups, and woody plants harbored many PPO genes. Eighteen PtrPPO genes were disproportionally localized on 19 chromosomes, and 3 pairs of segmented replication genes and 4 tandem repeat genomes were detected in poplars. Cis-acting element analysis identified numerous growth and developmental elements, secondary metabolism processes, and stress-related elements in the promoters of different PPO members. Furthermore, PtrPPO genes were expressed preferentially in the tissues and fruits of young plants. In addition, the expression of some PtrPPOs could be significantly induced by polyethylene glycol, abscisic acid, and methyl jasmonate, thereby revealing their potential role in regulating the stress response. Currently, we identified potential upstream TFs of PtrPPOs using bioinformatics.
\end{abstract}

\footnotetext{
*Correspondence: hefang1231@qq.com; w-xue@163.com

${ }^{\dagger}$ Fang He and Yu-Jie Shi contributed equally to this work.

Sichuan Province Key Laboratory of Ecological Forestry Engineering on the Upper Reaches of the Yangtze River, College of Forestry, Sichuan Agricultural University, Chengdu 611130, China
}

C C The Author(s). 2021 Open Access This article is licensed under a Creative Commons Attribution 4.0 International License, which permits use, sharing, adaptation, distribution and reproduction in any medium or format, as long as you give appropriate credit to the original author(s) and the source, provide a link to the Creative Commons licence, and indicate if changes were made. The images or other third party material in this article are included in the article's Creative Commons licence, unless indicated otherwise in a credit line to the material. If material is not included in the article's Creative Commons licence and your intended use is not permitted by statutory regulation or exceeds the permitted use, you will need to obtain permission directly from the copyright holder. To view a copy of this licence, visit http://creativecommons.org/licenses/by/4.0/. The Creative Commons Public Domain Dedication waiver (http://creativecommons.org/publicdomain/zero/1.0/) applies to the data made available in this article, unless otherwise stated in a credit line to the data. 
Conclusions: Comprehensive analysis is helpful for selecting candidate PPO genes for follow-up studies on biological function, and progress in understanding the molecular genetic basis of stress resistance in forest trees might lead to the development of genetic resources.

Keywords: Genome-wide, Populus, PPO gene family, Expression pattern, Stress

\section{Background}

Aggravated global warming induces more frequent and severe the local stress in whole ecosystems $[1,2]$. Plants have evolved complex mechanisms to defend themselves against various biotic and abiotic stresses [3, 4]. For example, plants frequently encounter insect pests and have evolved an efficient immune response that mainly depends on producing specific metabolites and defense proteins $[5,6]$. At the same time, plants competently transform their morphological structure, synthesize favorable metabolites, and evolve complex molecular mechanisms to deal with abiotic stresses [7-9], such as drought, cold, heat, and salt stress.

Polyphenol oxidase (PPO) is a widely distributed metalloproteinase that primarily exists in plants, fungi, and insects [10]. It can not only catalyze the oxidation of catechol to quinones but also acts on monophenol monooxygenase substrates. In a broad sense, PPO can be classified into three categories, i.e., tyrosinase (EC.1.14.18.1), catechol oxidase (EC.1.10.3.2), and laccase (EC.1.10.3.1) [11]. Of these three types of PPO, catecholase is mainly distributed in plants, while laccase and tyrosinase are distributed in microorganisms [12]. The function of PPO in plants is largely determined by three conserved domains i.e., KFDV, tyrosinase, and DWL [13].

PPO proteins predominantly exist in terrestrial plants, such as apple, litchi, spinach, potato, legume, tea, mulberry leaf, tobacco, and grapevine, as well as in fungi and some bacteria [11]. Under normal circumstances, PPO is inactive and tightly binds to the inner capsule membrane; activated PPO plays an important role after tissue damage. In addition, several PPO genes have been identified in Musa acuminata, Malus domestica, Oryza sativa, Sorghum bicolor, Solanum tuberosum, Ananas comosus, and Cucumis sativus [11, 14-19]. However, there are considerable differences in the distribution and function of PPO proteins in different plants. The location of PPO varies with plant species and maturity level; nevertheless, in most plant leaves, PPO is primarily distributed in chloroplasts [13], while almost all sub-cells of potato tubers contain PPO [12]. Polyphenol oxidase in tea exists in dissociative and combinative states; the former is chiefly found in the cytoplasm, while the latter is mainly found in chloroplasts, mitochondria, and other organelles $[11,20]$. Majority of studies have revealed that PPO activity and polyphenol content in tea shoots significantly affect the quality of red tea [21].
Extensive literature mining has revealed that the expression of PPO genes in plants is closely related to stress and response to insect and mechanical damage, diseases, and microorganism invasion [11, 22]. Additionally, the $P P O$ gene family exhibits a heightened response to methyl jasmonate (MeJA) in Salvia miltiorrhiza and Nicotiana tabacum $L$ [23, 24]. Moreover, overexpression of the $P P O$ gene in tomato can enhance plant resistance to insect pests, including Spodoptera litura, Helicoverpa armigera, and Spodoptera exigua [25, 26]. Similarly, the overexpression of $P P O$ genes in poplar trees can result in inhibition of the growth of Malacosoma disstria [27]. Furthermore, upregulation of $P P O$ expression in $\mathrm{Lyco}$ persicon esculentum, Juglans regia, Taraxacum officinale, and Fragaria ananassa enhances plant resistance to fungi $[11,25]$. Some key enzymes in the phenolic metabolic system play an essential role in mediating the resistance of plants to pathogenic microorganisms. For example, the pathogen-related protein $\mathrm{PPO}$ can catalyze the formation of lignin and quinones, thus promoting the formation of defensive barriers and protecting cells from invasion by strengthening the cell structure [28].

Populus has many advantages in basic research as a typical model woody plant with relatively upper ecological, economic, and cultural significance [29-31]. Stress severely restricts poplar cultivation in plantations. There are few functional studies on the role of the PPO protein family in biotic and abiotic stress in poplar trees. Here, we identified 18 putative PPO proteins in the Populus trichocarpa genome. Comparative genomics, transcriptomics, and RT-qPCR were used to comprehensively analyze the PPO protein family in poplar to provide a theoretical basis for studies on the characteristics and functions of PPOs in poplar development and stress response.

\section{Results}

\section{Identification and analysis of poplar PPOS}

Eighteen putative $P P O$ genes were identified from the published $P$. trichocarpa reference genome sequence and were successively designated PtrPPO1 to PtrPPO18 according to their location on the genome (Figure S1). In order to clearly understand the characteristics of the $P P O$ family in poplar, we analyzed the gene length, transcriptional sequence length, CDS (coding sequence) length, the position of the conserved domain, amino acid (AA) length, protein molecular weight (MW), grand 
average of hydropathicity (GRAVY), and isoelectric point (PI) of the proteins encoded by these genes (Table 1).

Among the 18 PtrPPO proteins, PtrPPO2 was the shortest with 192 amino acids, whereas PtrPPO9 was the longest (606 AA). The MW of PtrPPO proteins was 22.200 to $68.678 \mathrm{kDa}$, the GRAVY of the proteins was 0.821 (PtrPPO2) to -0.337 (PtrPPO11), and PI was 5.21 (PtrPPO4) to 7.53 (PtrPPO14). Generally, identifying the molecular characteristics of PPOs will be helpful in studying their specific biological functions.

Evolutionary and phylogenetic analysis of the PPO family To better understand the evolution and differentiation of PPO family proteins among species, 138 PPO amino acid sequences from 25 species were used to construct an unrooted tree (Fig. 1a). These sequences were then divided into 11 groups (I-XI), among which groups VIII and IX contained the most members, including 51 members accounting for $36.96 \%$ of the deduced PPO protein. PtrPPO protein was found in groups V, VIII, IX, and XI. Simultaneously, 14 PPO proteins in poplar were concentrated in group IX and clustered in the same branch as 7 PPO proteins in Salix purpurea.

To determine the origin and evolution of $P P O$ genes, we searched for $P P O$ genes in 25 species of lower aquatic plants and higher terrestrial plants (Fig. 1b). Interestingly, this gene was absent in Arabidopsis, but four $P P O$ genes were found in monocotyledonous $O$. sativa. It is worth noting that $P P O$ genes are widely distributed in woody plants, especially in $P$. trichocarpa
(18), M. domestica (15), and S. purpurea (10). However, Marchantia polymorpha (common liverwort, an herbaceous plant) contains $17 P P O$ genes. In conclusion, the distribution of $P P O$ genes in woody plants was much greater than that in herbaceous plants, indicating that considerable differentiation and doubling of $P P O$ genes might have occurred during the evolution of perennial woody plants.

\section{Determination of gene structure, conserved domains, and motifs}

To explore the evolutionary relationship between different members of the $P P O$ gene family more clearly, a phylogenetic tree was constructed using 18 PPO proteins from Populus (Fig. 2a), and their gene structure, conserved protein motifs, and conserved structure were assessed. Most PtrPPO genes (approximately two-thirds of the genes) had no introns, while the other six genes (PtrPPO2/3/5/11/14/17) had at most three introns. Surprisingly, none of the genes with two and three introns had a UTR (Fig. 2b). For example, PtrPPO7/8/1 genes lacked an intron, but they all had UTRs. However, PtrPPO2/5/14 contained at least two introns but no UTR.

Generally, genes with high similarity in amino acid sequences in the same family may have similar biological functions. We identified three conserved domains (PPO1-KFDV, tyrosinase, and PPO1_DWL) in the PPO protein sequence using $\mathrm{CDD}$ and SMART software (Fig. 2d and Table S3). All PPO proteins,

Table 1 Summary of Populus PPO genes

\begin{tabular}{|c|c|c|c|c|c|c|c|c|c|}
\hline Name & Gene model ID & gDNA & Transcript & CDS & Domains & AA & MW & GRAVY & $\mathrm{PI}$ \\
\hline PtrPPO1 & Potri.001G387900 & 1921 & 1921 & 1746 & $159-367 / 377-428 / 453-578$ & 581 & 64.913 & -0.453 & 6.00 \\
\hline PtrPPO2 & Potri.001G388000 & 662 & 579 & 579 & 5-44/47-97/110-189 & 192 & 22.200 & -0.821 & 5.74 \\
\hline PtrPPO3 & Potri.001G388100 & 2552 & 1584 & 1566 & $91-307 / 317-368 / 393-518$ & 521 & 58.651 & -0.413 & 5.35 \\
\hline PtrPPO4 & Potri.001G388200 & 1927 & 1927 & 1746 & $159-367 / 377-428 / 453-578$ & 581 & 64.757 & -0.436 & 5.21 \\
\hline PtrPPO5 & Potri.001G388300 & 2009 & 1326 & 1326 & $74-227 / 237-287 / 313-438$ & 441 & 49.561 & -0.527 & 5.28 \\
\hline PtrPPO6 & Potri.001G388400 & 1752 & 1752 & 1746 & $159-367 / 377-428 / 453-578$ & 581 & 64.999 & -0.449 & 5.96 \\
\hline PtrPPO7 & Potri.001G388600 & 1805 & 1805 & 1746 & $159-367 / 377-428 / 453-578$ & 581 & 64.667 & -0.424 & 5.68 \\
\hline PtrPPO8 & Potri.001G388800 & 1940 & 1940 & 1746 & $159-367 / 377-428 / 453-578$ & 581 & 65.008 & -0.449 & 6.14 \\
\hline PtrPPO9 & Potri.001G388900 & 2149 & 2149 & 1821 & $193-400 / 407-455 / 482-603$ & 606 & 68.678 & -0.450 & 6.53 \\
\hline PtrPPO10 & Potri.004G038500 & 1346 & 1346 & 744 & $118-244$ & 247 & 27.230 & -0.515 & 6.93 \\
\hline PtrPPO11 & Potri.004G156500 & 2303 & 2140 & 1764 & $140-357 / 363-413 / 453-585$ & 587 & 67.459 & -0.337 & 6.36 \\
\hline PtrPPO12 & Potri.011G047200 & 1058 & 1058 & 732 & $115-240$ & 243 & 26.419 & -0.419 & 6.21 \\
\hline PtrPPO13 & Potri.011G047300 & 2135 & 2135 & 1773 & $160-368 / 374-425 / 460-587$ & 590 & 66.288 & -0.536 & 6.55 \\
\hline PtrPPO14 & Potri.011G108200 & 3295 & 1713 & 1713 & $184-348 / 358-408 / 430-522$ & 570 & 64.747 & -0.516 & 7.53 \\
\hline PtrPPO15 & Potri.011G108300 & 2013 & 2013 & 1692 & $145-351 / 361-411 / 433-560$ & 563 & 64.066 & -0.514 & 5.96 \\
\hline PtrPPO16 & Potri.T061900 & 1921 & 1914 & 1746 & $159-367 / 377-428 / 453-578$ & 581 & 65.077 & -0.455 & 6.23 \\
\hline PtrPPO17 & Potri.T062100 & 1889 & 1688 & 1638 & $159-331 / 341-392 / 417-542$ & 545 & 60.654 & -0.420 & 5.86 \\
\hline PtrPP018 & Potri.T062200 & 1947 & 1947 & 1746 & $159-367 / 377-428 / 453-578$ & 581 & 64.889 & -0.442 & 6.30 \\
\hline
\end{tabular}




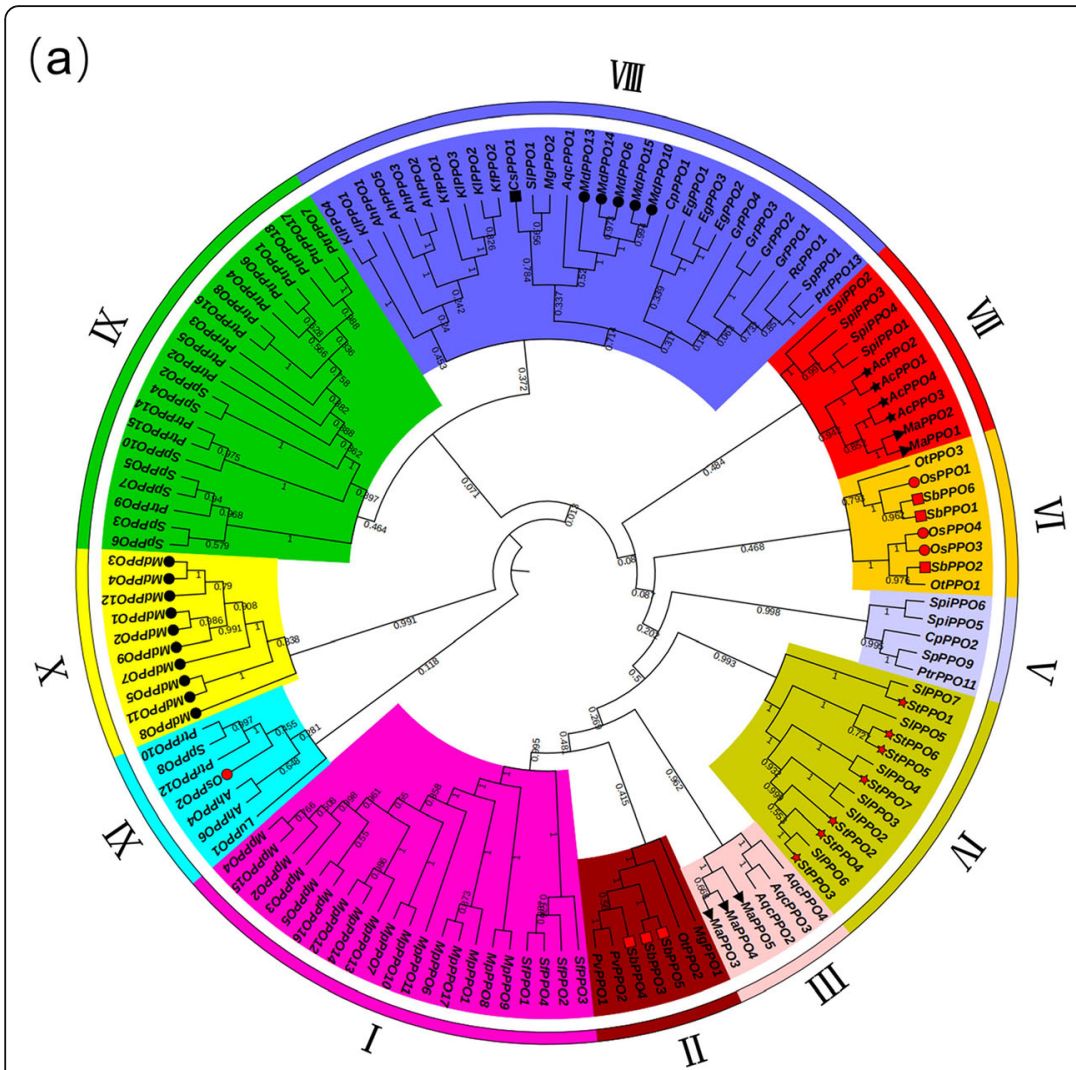

(b)

Fig. 1 Evolutionary and phylogenetic analysis of the PPO family in diverse plant species. a The phylogenetic tree of PPO proteins from Aquilegia coerulea (AqcPPO), Ananas comosus (AcPPO), Amaranthus hypochondriacus (AhPPO), Carica papaya (CPPPO), Cucumis sativus (CsPPO), Eucalyptus grandis (EgPPO), Gossypium raimondii (GrPPO), Kalanchoe fedtschenkoi (KfPPO), Kalanchoe laxiflora (KIPPO), Linum usitatissimum (LuPPO), Musa acuminata (MaPPO), Malus domestica (MdPPO), Mimulus guttatus (MgPPO), Marchantia polymorpha (MpPPO), Oryza sativa (OsPPO), Oropetium thomaeum (OtPPO), Populus trichocarpa (PtrPPO), Panicum virgatum (PVPPO), Ricinus communis (RCPPO), Sorghum bicolor (SbPPO), Sphagnum fallax (SfPPO), Solanum lycopersicum (SIPPO), Spirodela polyrhiza (SpiPPO), Salix purpurea (SPPPO), and Solanum tuberosum (StPPO). The shape and color in front of the node represent the identified PPOs, in which the black circle represents MdPPOs, the black rectangle represents CSPPOs, the black pentagram represents ACPPOs, the black triangle represents MaPPOs, the red circle represents OSPPOS, the red rectangle represents SbPPOs, and the red pentagram represents StPPOs. b Comparisons of PPO protein numbers across 25 plant species. The accession numbers and gene names are all shown in Table S2

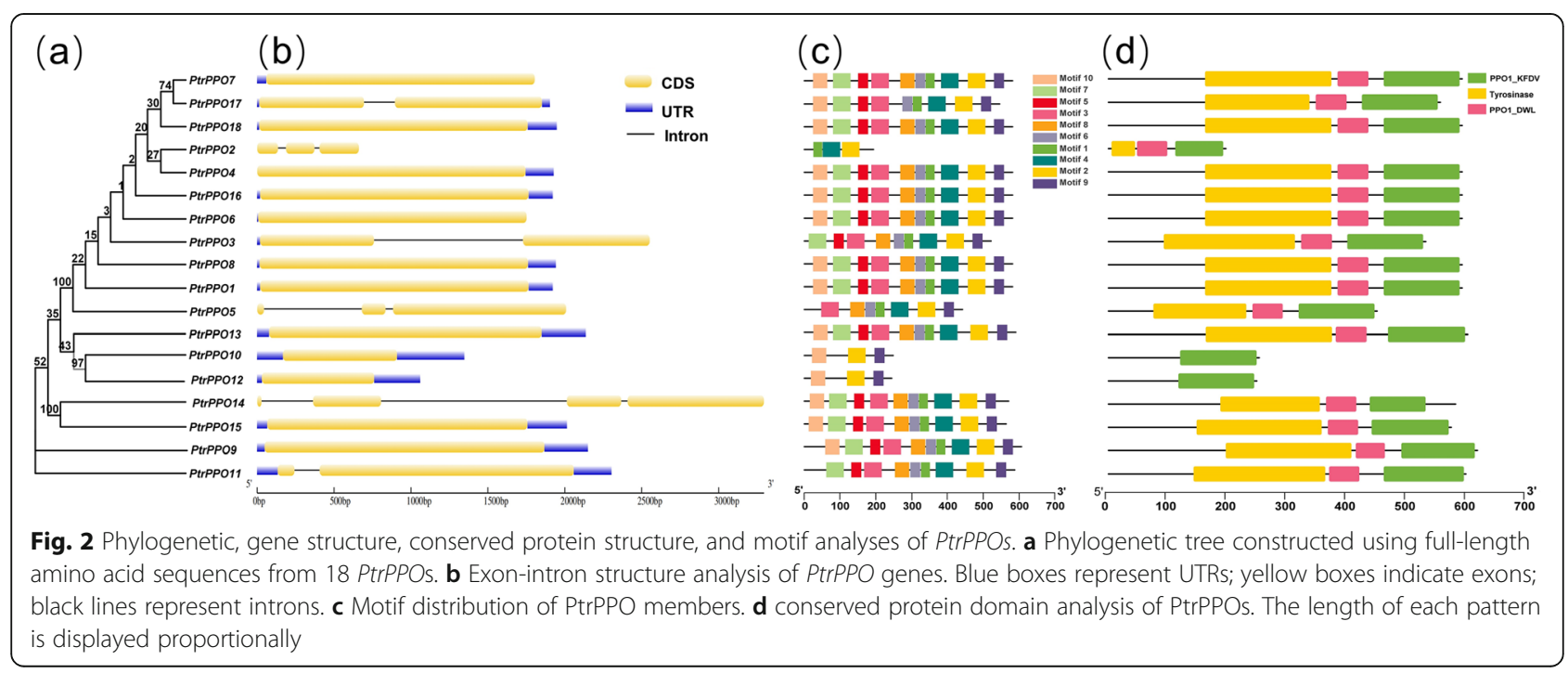


except for PtrPPO10/12, had these three structures, which may endow them with similar biological functions. Meanwhile, 10 conserved motifs were identified in the PtrPPOs protein sequence using MEME (Fig. 2c). Amino acid sequence encode, and SeqLogo of the Motif are displayed in Table S4. Among them, two motifs (2 and 9) were related to the C-terminal PPO-KFDV domain, motif 4 was related to the intermediate PPO_DWL domain, and five motifs $(1,6,8$, 3 , and 5) were related to the $\mathrm{N}$-terminal tyrosinase domain. Moreover, we found two novel motifs (motifs 7 and 10) at the N-terminal of most PtrPPO protein sequences.

\section{Analysis of chromosomal location and gene duplication}

To further understand the evolution and differentiation of $P P O$ family genes, we analyzed the chromosomal distribution, synteny, and evolution of the 18 PPO genes in Populus. The PPO genes were primarily distributed on chromosomes 1, 4, 11, and scaffold_64 (Fig. 3 and Figure $\mathrm{S} 1)$. Moreover, half of the $P P O$ genes in poplar were distributed on chromosome 1, while there were, at least, two $P P O$ genes on the other chromosomes. It has been reported that the chromosome region within $200 \mathrm{~kb}$ containing two or more genes is defined as a tandem replication event [32]. The 18 genes (PtrPPO1/2/3/4/5/ 6/7/8/9, PtrPPO12/13, PtrPPO14/15, PtrPPO16/17/18) formed four tandem repeat regions distributed on chromosomes 1, 11, and scaffold_64 (Fig. 3). The high sequence similarity between repetitive gene pairs indicates that they are likely involved in regulating similar biological processes. Furthermore, nine PPO genes formed a complex tandem repeat on chromosome 1, indicating a hot spot for $P P O$ gene distribution. We also found that six genes (PtrPPO1/14, PtrPPO8/15, PtrPPO10/12) formed three segmental duplication events using the MCScanX method. These results suggest that PtrPPOs might be produced by gene replication, while tandem duplication and repetitive fragment events collectively catalyze the evolution of $P P O$ genes in poplar.

We constructed comparative syntenic maps between poplar and four other species to analyze PPO gene evolution in woody plants further. Syntenic maps revealed that nine pairs of homologous genes were found

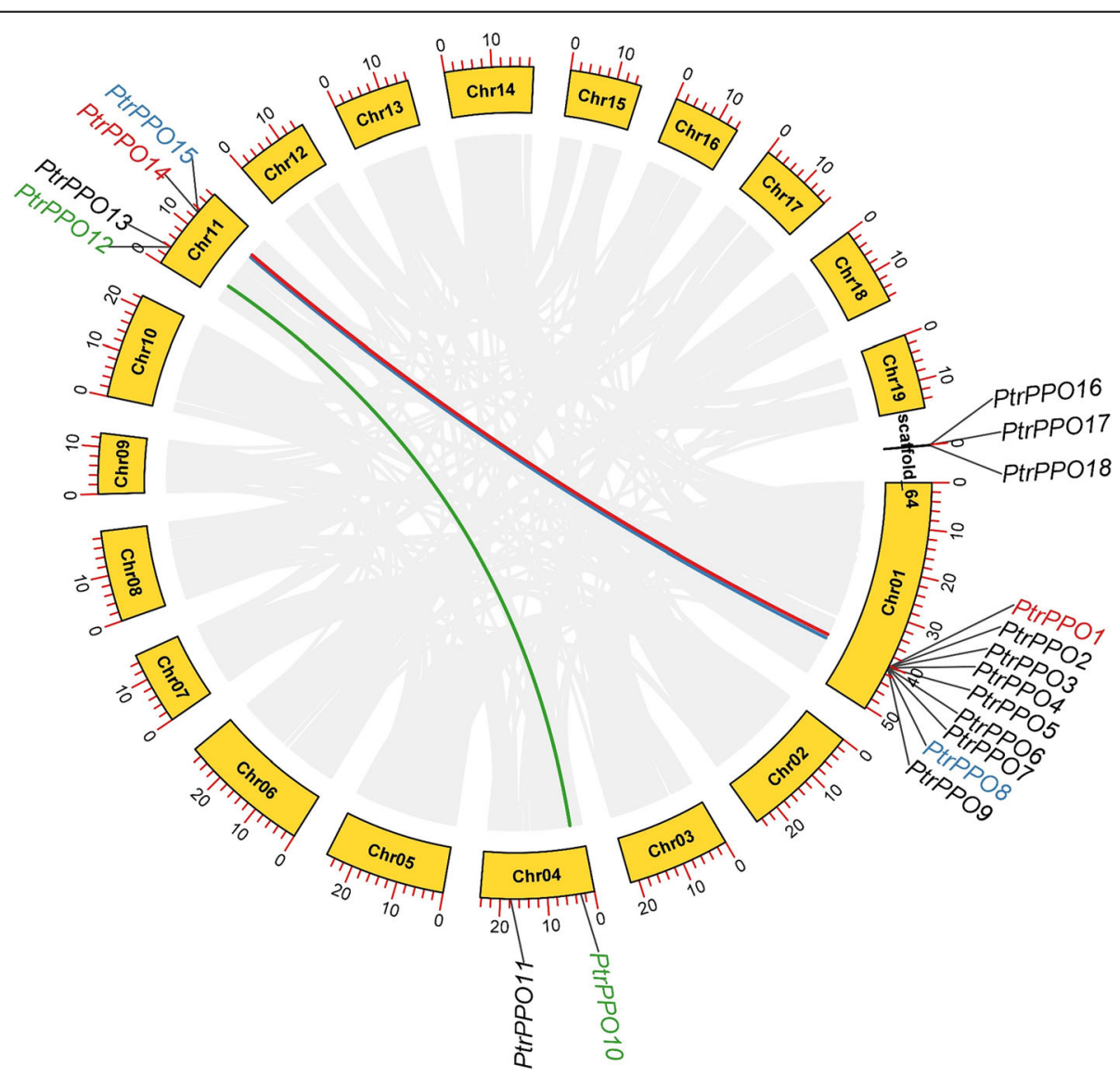

Fig. 3 Schematic diagram depicting PPO gene distribution and Inter-chromosomal relationships in Populus chromosomes. The gray lines represent all synteny blocks in the chromosome of Populus trichocarpa. Red, blue, and green lines indicate duplicated PPO gene pairs in Populus trichocarpa. Chromosome number is shown in the middle of the arc square. The length of each arc corresponds to the length of the chromosome (Mb) 
between $P$. trichocarpa and $S$. purpurea, and three pairs of homologous genes were found between $P$. trichocarpa and $M$. domestica (Fig. 4 and Table S5). Some PtrPPO genes (PtrPPO10 and PtrPPO12) were discovered to be connected with at least two synonym gene pairs (especially between poplar and willow, and between poplar and apple $P P O$ genes), suggesting that these genes may play essential roles in the evolutionary process of the PPO gene family. However, the findings of previous studies and those of the present study revealed that there was no synteny in Populus vs. O. sativa and Populus vs. A. thaliana [11], suggesting that multiple PPO genes may be formed during the differentiation of woody plants.

\section{PtrPPO cis-element analysis}

To determine the expression pattern of PtrPPOs, the TSS promoter-upstream region $(\sim 2000 \mathrm{bp})$ sequences were extracted from the genomic DNA sequence of $P$. trichocarpa. The cis-elements of the PtrPPOs promoter were analyzed using the PlantCARE database (Fig. 5). The specific functions of these motifs (cis-elements) are annotated in Table S6. A series of cis-acting elements involved in all aspects of poplar development, including palisade mesophyll cell differentiation, seed-specific regulation, cell cycle regulation, endosperm expression, meristem expression, and gibberellin-responsive and auxin-responsive elements, were identified, indicating the crucial role of PtrPPOs in poplar development. For example, the promoter regions of many $P P O$ genes were identified to contain gibberellin-responsive elements, and the PtrPPO5 promoter contained three gibberellinresponsive elements, indicating that this gene is likely involved in gibberellin-signal transduction (Table S7). Moreover, the promoter region of PtrPPO10 contains elements of cell cycle regulation, meristem expression, and endosperm expression, indicating that this gene may be involved in the regulation of poplar growth and development.

Furthermore, PtrPPO promoter regions were associated with secondary metabolism processes, including zein metabolism regulation and flavonoid biosynthetic gene regulation. PtrPPO8 contained elements related to the aforementioned processes, suggesting that this gene might be involved in secondary metabolism in Populus. Besides, some stress-related elements were present in the promoter region, including MeJA responsiveness, salicylic acid responsiveness, abscisic acid

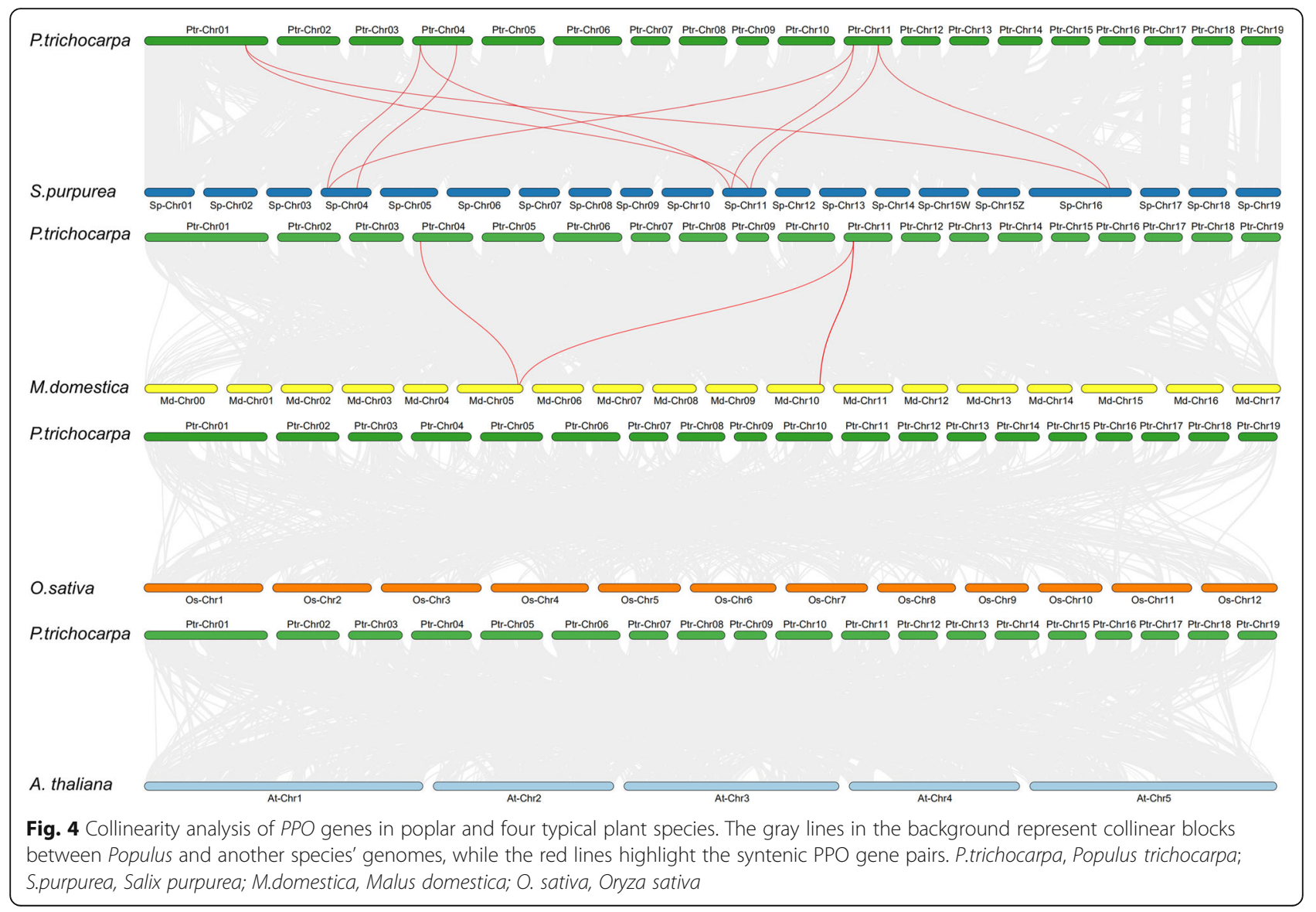




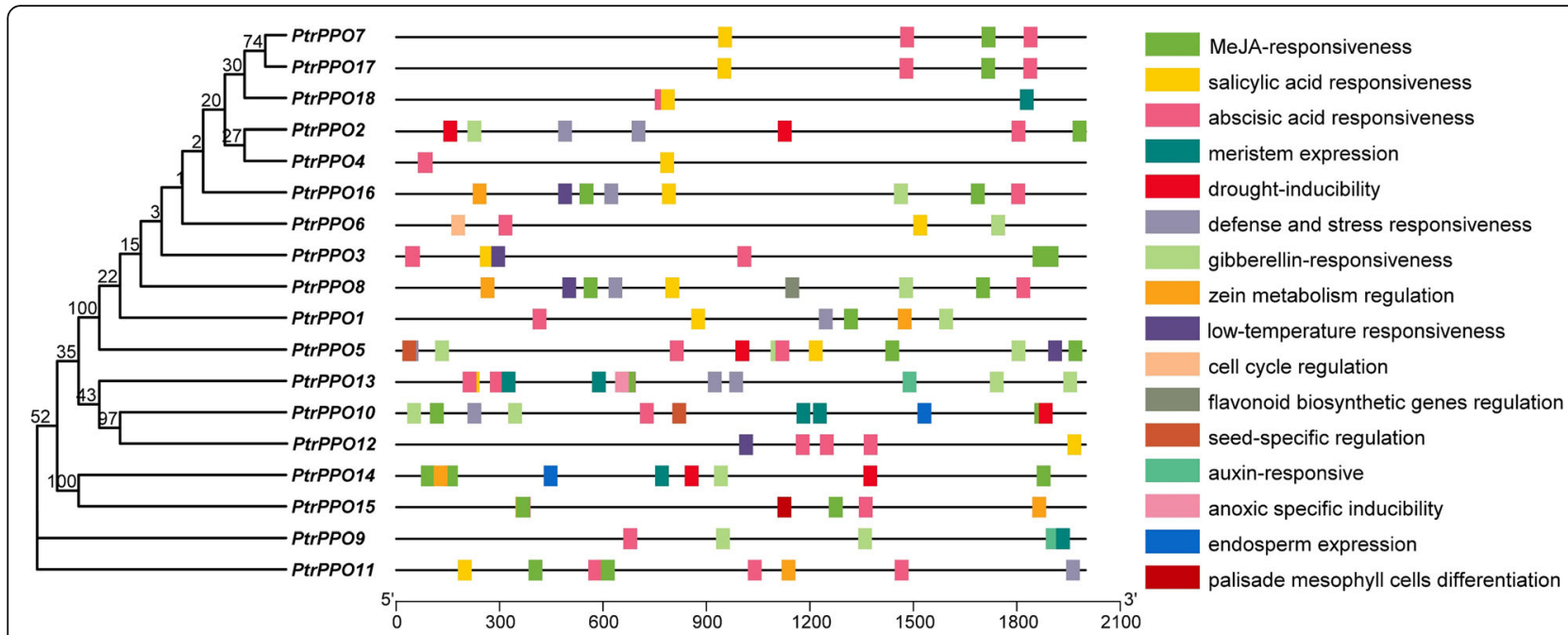

Fig. 5 Cis-element analysis of the PtrPPOs. The relative locations of stress and growth-related cis-elements in the PtrPPO promoter region. Different colors represent different cis-acting elements, and their position corresponds to the corresponding position of the promoter

responsiveness, drought inducibility, defense and stress responsiveness, anoxic specific inducibility elements, and low-temperature responsiveness, which implied that PtrPPOs might also respond to stress. Except for PtrPPO4/6/9/12/18, all other $P P O$ genes contained MeJA-responsive elements (Table S7), indicating that most $P P O$ genes were involved in MeJA signal transduction. Moreover, PtrPPO13 harbored multiple elements associated with stress, including two MeJA-responsive elements, one salicylic-acid-responsive element, four abscisic-acid-responsive elements, and two defense and stress responsive elements (Table S7), indicating that PtrPPO13 may respond to both biological and abiotic stresses.

\section{RNA-seq analysis of Populus PPO genes}

To explore the role of $P P O$ genes in poplar growth and development, we analyzed the tissue expression patterns of 18 PtrPPOs in the transcriptomic dataset. First, we found that PtrPPO2 was not detected in all tissues and that it might be a pseudogene or might have a specific spatiotemporal expression pattern (not included in the library). Next, we constructed a dual clustering heat map (sample and gene) to investigate the expression profile of these genes in 15 Populus tissues (Fig. 6a and Table S8). Interestingly, some genes were preferentially expressed in specific plant tissues. Eight genes were highly expressed in young expanding leaves (PtrPPO14/ $15 / 18 / 3 / 7 / 16 / 4 / 5)$, two genes in whole suckers and freshly expanding leaves (PtrPPO14/15), five genes in dormant and prechilled buds (PtrPPO18/3/7/4/5), and one gene in mature seeds (PtrPPO9). It was further revealed that most of these genes were found in young plant tissues or seeds. For example, PtrPPO18 was expressed at high levels in young tissues but at low levels in mature tissues (such as mature leaves and petioles).

To further understand the role of $P P O$ genes in the response of poplar plants to stress, we mapped the expression configurations of the 18 poplar $P P O$ genes following exposure to drought and beetle- and mechanical-damage using the uploaded transcriptome dataset (Fig. 6b and Table S9). The heat map revealed that the expression of PtrPPO9 was significantly induced in leaves in response to drought stress, and all three treatments significantly induced the expression of PtrPPO13. However, the expression of PtrPPO11/14/15 was significantly inhibited under conditions of beetleand mechanical-damage-induced stress. These results will be helpful for future research on gene function.

\section{Expression profile of Populus PPO genes in different plant tissues and treatments}

The level of $P P O$ gene expression in plants is closely related to stress and primarily reflects disease, insect and mechanical damage, and microorganism invasion [11, 22]. Results showed that the expression of five genes, i.e., PtrPPO9/11/13/14/15, was significantly induced or inhibited under conditions of stress. To verify the reliability of $P P O$ gene expression in the transcriptome, the expression of 5 PPO (PtrPPO9/11/13/14/15) genes was investigated using RT-qPCR (Fig. 7a). Consistent with the previous results, most genes were expressed in young tissues. For example, except for PtrPPO13, which was preferentially expressed in mature leaves, the other four genes (PtrPPO9/11/14/15) were expressed primarily in the young leaves. Notably, PtrPPO9/11/13/14/15 were expressed at high levels in leaves (compare to other tissues, such as xylem, phloem, and root). 

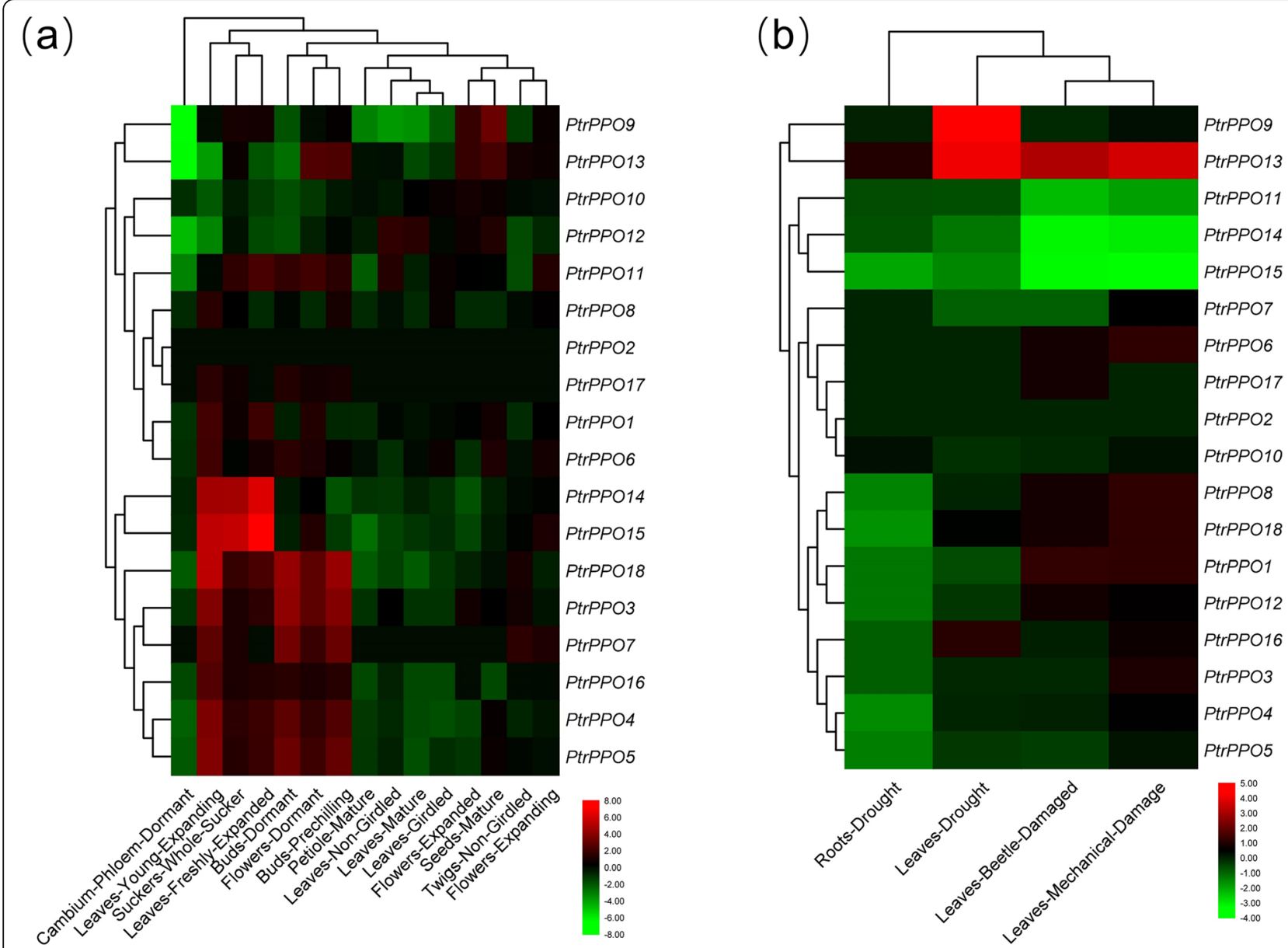

Fig. 6 Expression profile of PPO genes under developmental and stress conditions. a Heat maps showing the expression levels of 18 PtrPPO genes in different tissues at different developmental stages based on transcriptome data. $\mathbf{b}$ Heat maps depicting the expression levels of 18 PtrPPO genes following drought-, beetle-, and mechanical damage. The color bar represents the range of maximum and minimum values of relative expression in the heat map

To further validate whether $P P O$ abundance was affected by abiotic stress and hormone treatment, the expression of 5 PPO members-from among the 18 PtrPPOs genes-was carefully investigated in response to PEG, ABA, and MeJA treatment using qRT-PCR (Fig. $7 \mathrm{~b}-\mathrm{d})$. When poplar plants were treated with PEG, the expression of the other four genes was significantly induced except PtrPPO11 (Fig. 7b). PEG treatment significantly upregulated the expression of PtrPPO9/13 that peaked $6 \mathrm{~h}$ after treatment. PtrPPO14 was significantly upregulated after $3 \mathrm{~h}$ of treatment, and its expression level remained at approximately 2 -fold. Furthermore, PtrPPO15 was upregulated following PEG treatment, with a maximum expression of approximately 8-fold (compared to pre-treatment levels) after $9 \mathrm{~h}$.

In response to ABA treatment, PtrPPO9/13 showed similar expression patterns, with the expression of both genes significantly induced in response to ABA treatment, peaking after $3 \mathrm{~h}$ of treatment (approximately 20- and 15-fold pre-treatment levels, respectively) (Fig. 7c). However, the expression of PtrPPO11/14/15 was significantly inhibited by ABA. In response to MeJA treatment, the expression of PtrPPO13 was significantly induced, reaching a maximum value approximately 5 -fold that of pre-treatment expression levels at $6 \mathrm{~h}$ of treatment, decreasing to approximately 3 -fold at $9 \mathrm{~h}$ of treatment (Fig. $7 d)$. In contrast, the expression of PtrPPO11/14/15 was significantly inhibited by MeJA, whereas that of PtrPPO9 was induced at 1 and $3 \mathrm{~h}$ but significantly inhibited at 9 and $12 \mathrm{~h}$. Overall, each treatment may differentially induce and/or inhibit the expression of PPO genes in Populus.

\section{Identification and expression pattern analysis of potential upstream transcription factors (TFs) of PtrPPOs}

To further understand how PtrPPO9/11/13/14/15 function under stressful conditions, we identified potential upstream TFs of PtrPPOs using bioinformatics (Table 


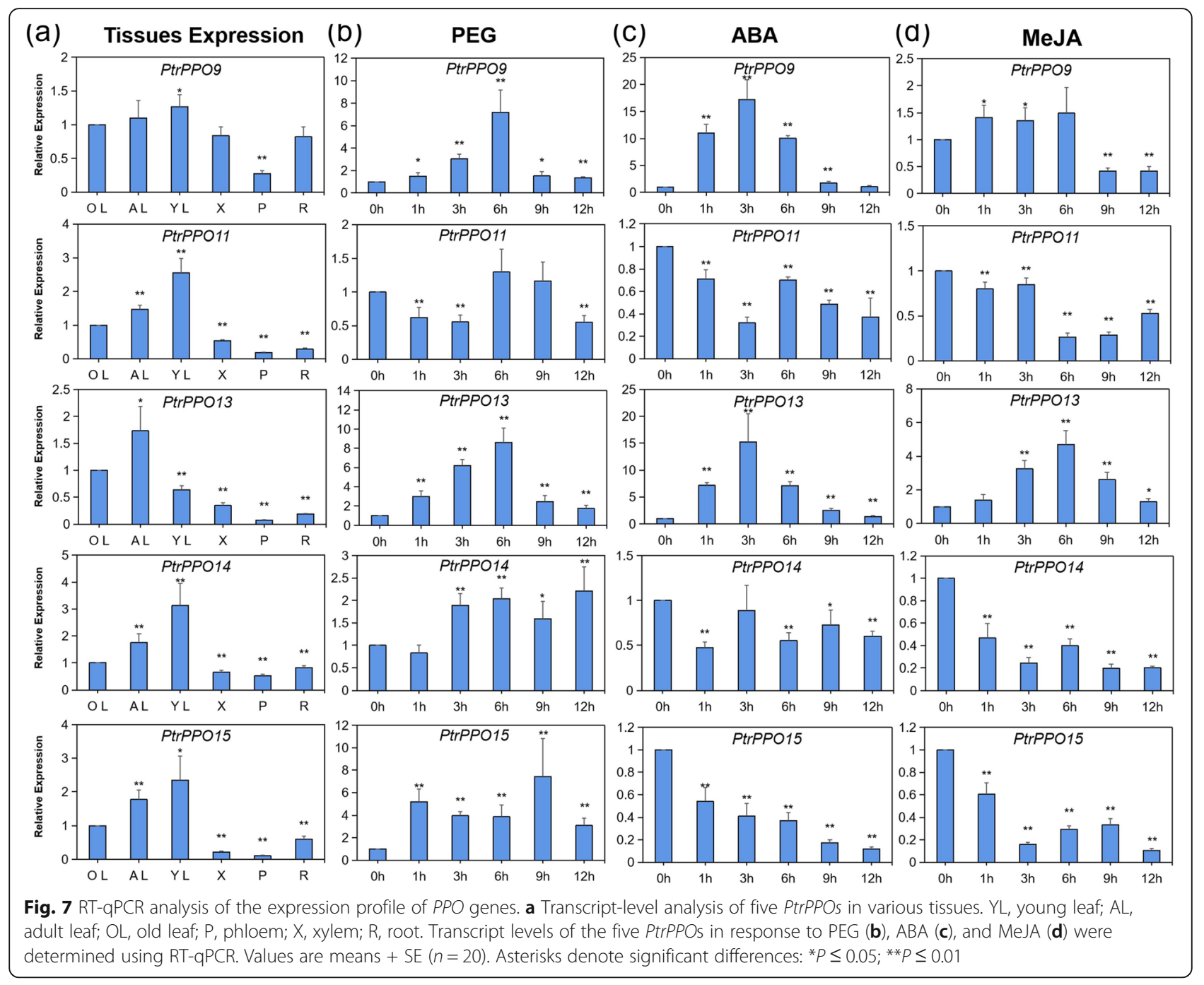

S10). The regulatory network generated using Cytoscape (Fig. 8a) revealed that the expression of PtrPPO9/11/13/ $14 / 15$ might be regulated by $10,7,19,20$, and $1 \mathrm{TFs}$, respectively. These TFs include Dof, ERF, BBR-BPC, MIKC_MADS, AP2, LBD, ARF, bHLH, B3, C2H2, GRAS, GATA, MYB, Nin-like, SBP, TALE, and WRKY family proteins. Moreover, Potri.002G026700, Potri.002G151700, Potri.010G181000, and Potri.014G074200 may simultaneously regulate the expression of PtrPPO9/13/14 genes. We also found that Potri.010G101400 may regulate the expression of three genes PtrPPO13/14/15.

We simultaneously evaluated the expression pattern of potential upstream TFs of PtrPPO9/11/13/14/15 under stress conditions (Fig. 8b-f and Table S11). The potential upstream MIKC_MADS TFs (Potri.002G151700) and bHLH TFs (Potri.003G207200) of PtrPPO9 were significantly upregulated in response to drought, beetle-, and mechanical-damage in poplar leaves (Fig. 8b). In addition, the expression of Potri.003G034200 (Dof family protein), Potri.009G064700 (bHLH family protein), and Potri.004G056900 (Dof family protein) was significantly induced in beetle-damaged leaves (Fig. 8b). When poplar trees were exposed to beetle- and mechanical-damage, the expression of potential upstream ARF TFs (Potri.005G236700 and Potri.012G106100) of PtrPPO11 was significantly induced, and that of the potential upstream Dof TFs (Potri.001G238400 and Potri.007G036400) of PtrPPO11 was significantly inhibited (Fig. 8c).

Exposure of poplar trees to beetle- and mechanicaldamage-induced stress resulted in the significant downregulation of the potential upstream TALE (Potri.002G113300), bHLH (Potri.007G023600), Dof (Potri.007G036400), and B3 TFs (Potri.001G322700) of PtrPPO13 (Fig. 8d). Moreover, the expression of the potential upstream TFs of PtrPPO14 was significantly induced by stress, except for ERF TFs (Potri.018G028000) (Fig. 8e). The expression of potential upstream BBR-BPC TF (Potri.010G101400) of PtrPPO15 was significantly 


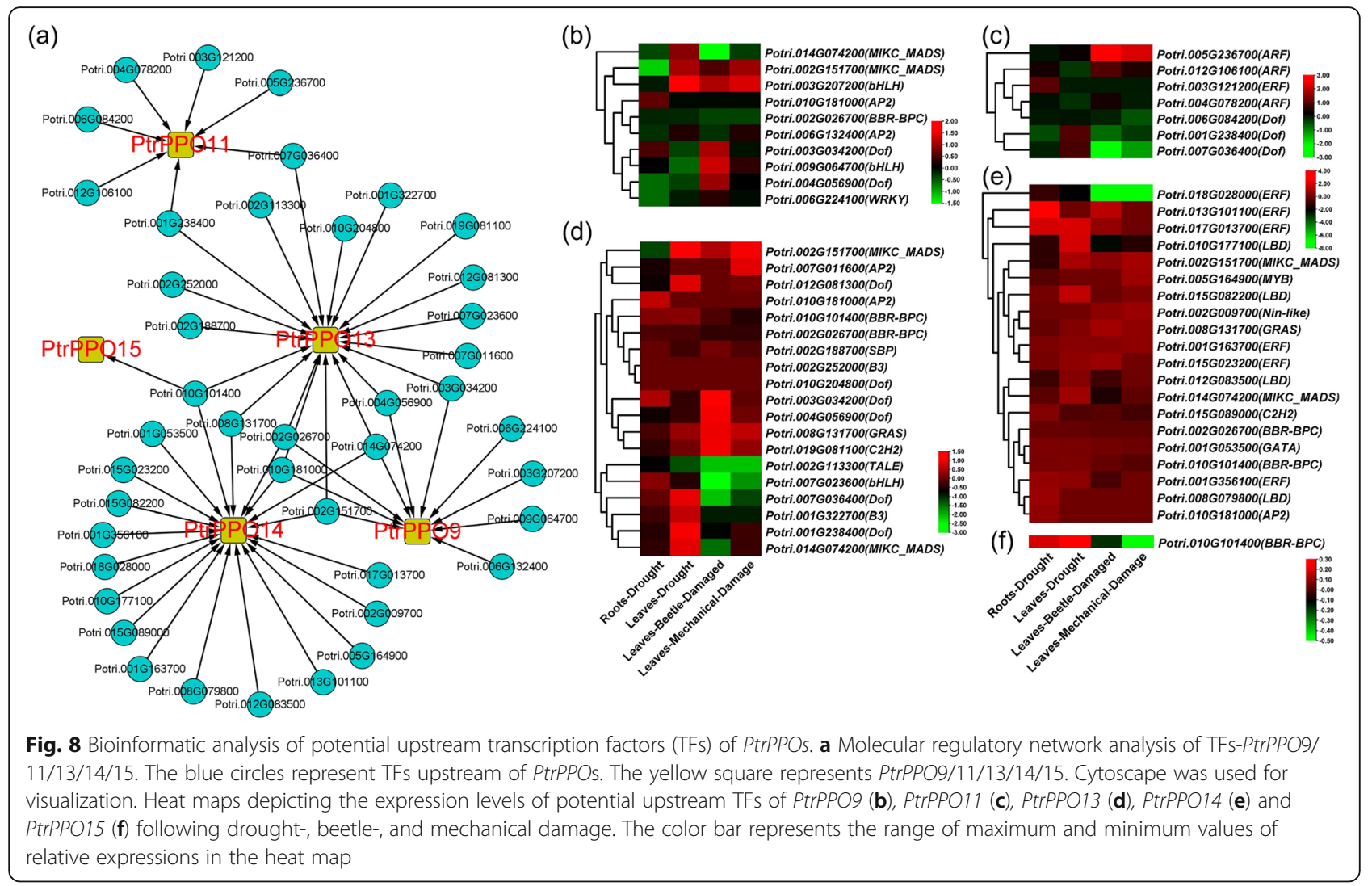

upregulated by drought stress in poplar leaves; however, it was significantly inhibited by beetle- and mechanicaldamage induced stress (Fig. 8f).

\section{Discussion}

As widespread copper metalloenzymes in plants, PPOs play an important role in plant growth, development, and stress tolerance [10, 20, 24]. With the development of whole genome sequencing, an increasing number of $P P O$ genes have been identified in different plants, including apple, litchi, opium, potato, legumes, tea, mulberry, and tobacco [11]. However, the PPO family in the woody poplar plants is yet to be fully understood.

In the present study, $18 P P O$ genes were identified in poplar plants, and the characteristics of all products after replication, transcription, and translation were analyzed; additionally, their systematic evolution and expression models were constructed. In line with previous gene family studies [32], $P P O$ genes in poplars were designated PtrPPO1 to PtrPPO18 according to their chromosomal locations (Table 1 and Figure S1). Except for PtrPPO10/12, all PtrPPO proteins had three conserved domains (KFDV, tyrosinase, and DWL), which may endow them with similar biological functions. It has been reported that the loss of protein structure could lead to different gene functions [33]. As both PtrPPO10 and PtrPPO12 only had one KFDV domain (Fig. 2d), they were clustered in the same branch (Fig. 1a and 2a), and the length of gDNA and the number of motifs in the protein were significantly lower than those in other genes (Fig. 2c). Furthermore, PtrPPO10 and PtrPPO12 only contained the PPO1_KFDV domain (Fig. 2d), suggesting the two proteins may have similar functions. As shown above, PtrPPO10 and PtrPPO12 cluster together when tissue expression patterns are investigated (Fig. 6a). The two genes have similar tissue expression patterns, and both are expressed at high levels in flowers and seeds, indicating that they may play a role in the formation of poplar flowers and seeds.

Most PtrPPOs have no introns, whereas some have three introns at most (Fig. 2b). Research has shown that introns play essential roles in the regulation of the transcriptome [34]. In order to respond quickly to stress, organisms need to stimulate the expression of genes, and gene structures with a few or no introns contribute to the rapid expression of mRNA [35]. For example, PtrPPO7/8/1 genes lack introns, but contain UTRs; therefore, they can transcribe faster to form mRNA. In many plants, PPO genes respond quickly to both biological and abiotic stresses [11, 24].

We constructed an evolutionary tree from 25 model plants and divided it into 11 sections to further determine the origin and evolution of $P P O$ genes. Consistent with the aforementioned results (Fig. 2a), PtrPPO 
protein was found in Groups V, VIII, IX, and XI (Fig. 1a). The phylogenetic tree analysis of family genes can clearly describe the evolutionary course of genes [36]. Because Populus (poplar) and Salix (willow) are both members of the family Salicaceae [37], most of their $P P O$ genes are clustered in the same branch (IX). Notably, the $P P O$ gene was not found in Arabidopsis, but four $P P O$ genes were found in monocotyledonous $O$. sativa (Fig. 1b). It is worth noting that $P P O$ genes are widely distributed in woody plants, especially in $P$. trichocarpa (18), M. domestica (15), and S. purpurea (10). No gene pairs have been found between Arabidopsis and poplar, whereas multiple gene pairs have been found between apple and poplar and between S. purpurea and poplar (Fig. 4), indicating that considerable differentiation and doubling of PPOs might have taken place during the evolution of perennial woody plants.

The increase in gene family members and the mechanism of genome evolution primarily depend on gene replication events, including tandem and segmented replications [38]. In the current study, 18 PtrPPO genes were unevenly distributed on 19 poplar chromosomes, and half of these PtrPPO genes were located on chromosome 1. This phenomenon of uneven distribution of chromosomes indicates that the change occurred before species differentiation. A total of three pairs of segmented replication genes and four tandem repeat genomes were detected in poplars, indicating that both tandem and segmental repeats contribute to the evolution of PtrPPO genes in Populus (Fig. 3). Previous gene family studies have shown that tandem repeat genes may have similar functions and expression patterns [34, 39]. For example, PtrPPO12/13 has a similar expression level in all tissues, while PtrPPO14/15 show a downward trend under adverse conditions (Fig. 6). Similar expression levels indicate similar functions and structures of tandem repeat genes.

The expression pattern of $P P O$ genes in various tissues has been demonstrated in numerous species [22, 27, 28, 40]. Due to the difference in the number of PPOs in different species, there are no uniform gene expression profiles of $P P O$ genes in plants. According to the RNAseq data of poplar, some genes are preferentially expressed in young plant tissues and seeds (Fig. 6a), suggesting these tissues are more attractive to intruders. Consistent with the above results, fluorescence quantitative PCR results also showed that the PPO was expressed preferentially in young leaves than in other tissues (Fig. 7a). Research elsewhere has indicated that young leaves have higher PPO activity than old leaves [41].

The expression of $P P O$ genes in plants is closely related to stress and mainly responds to insect and mechanical damage, diseases, and microorganism invasion $[11,22]$. Transcriptome dataset analysis revealed that some $P P O$ genes in poplar were significantly induced
(PtrPPO 9/13) and inhibited (PtrPPO 11/14/15) by drought. A similar trend was observed using RT-qPCR. PEG and ABA treatments significantly upregulated PtrPPO 9/13 expression (Fig. 7b and c). By analyzing the promoters of PtrPPO 9/13, we found that there were multiple abscisic acid responsiveness elements in these two promoters (Fig. 5). PtrPPO9 was significantly upregulated under conditions of PEG and ABA treatment than under MeJA treatment, indicating that PtrPPO9 had a higher probability of being induced by abiotic stress. In addition, PPO genes in Nicotiana tabacum and Glycine max were significantly induced by ABA [24, 42] . Transcriptome analysis verified that PtrPPO13 was significantly upregulated, and PtrPPO11/14/15 was significantly inhibited in response to mechanical injury and damage caused by the poplar borer (beetle; Fig. 6b). MeJA is involved in signal transduction during insect attack and plant mechanical damage [23, 24]. MeJA responsiveness elements were identified on the PtrPPO13 promoter. Additionally, RT-qPCR revealed that PtrPPO13 was significantly induced by MeJA (Fig. 7d). PtrPPO13 was significantly induced by PEG, ABA, and MeJA treatments, reaching a peak at 3 or $6 \mathrm{~h}$, suggesting that PtrPPO13 might be involved in biological and abiotic stress. Compared with the expression profiles revealed by RNA-seq data, the patterns of qRT-PCR expression were dissimilar. Various reasons might cause the observed differences in expressions.

In recent years, promoters, TFs, and microRNAs (miRNAs) have been found to regulate $P P O$ response to stress in plants [11]. miR528, miR12112, and miR058 from Banana, Salvia miltiorrhiza, and Grapevine can silence PPO genes and participate in cold stress and MeJA response, respectively [23, 43-45]. Similarly, TFs play a crucial role in the transcriptional regulation of genes [46]. The potential upstream TFs of PtrPPOs were identified using bioinformatics (Fig. 8a). Compared with the previous results (Fig. 6b), some potential upstream TFs of PtrPPOs were also induced significantly in stress (Fig. 8a-f). Recently, it has been reported that MnMYB3R1 in Mulberry can combine with the MSA element on the MnPPO1 promoter and then regulate $M n P P O 1$ to increase plant drought resistance [28]. In conclusion, PPOs play a critical role in the regulation of stress response.

The finding of the current study allows us to infer the functional roles of $P P O$ genes in poplar plants. Comprehensive analysis is useful to select candidate $P P O$ genes for further functional characterization, while genetic improvement of stress resistance in forest trees provides genetic resources.

\section{Conclusions}

To summarize, whole-genome analysis of the poplar PPO family was accomplished, and 18 PtrPPO genes 
were identified. Bioinformatics and qRT-PCR were then used to analyze the gene structure, phylogeny, chromosomal localization, gene replication, cis-elements, and expression patterns of PtrPPOs. Finally, we found that $P P O$ genes were preferentially expressed in young plant tissues and fruits, and some genes could be significantly induced by PEG, ABA, and MeJA, indicating that PPO plays an integral part in the stress resistance of poplars. This research will help to explore the function of $P P O$ genes in Populus.

\section{Methods}

Genome-wide identification and retrieval of PPO genes in P. trichocarpa

The extraction and identification of poplar family members were conducted according to a previously described method [47]. The genome data of $A$. thaliana (Athaliana_447_TAIR10.fa.gz), $O$. sativa (Osativa_323_ v7.0.fa.gz), and P. trichocarpa (Ptrichocarpa_533_ v4.0.fa.gz) were obtained from the Phytozome database (https://phytozome.jgi.doe.gov/pz/portal.html) [48]. In addition, BLAST and HMMER were used to identify 18 PPO proteins with conserved structures in poplar plants. Redundant sequences were manually discarded. Furthermore, the conservative structure, molecular weights, isoelectric points, and hydrophilicity analysis of the identified PPOs were conducted using SMART on the ExPasy server (https://web.expasy.org/protparam/) [32].

\section{Evolutionary relationships of $P P O$ genes}

A total of 138 PPO proteins from Aquilegia coerulea, Ananas comosus, Amaranthus hypochondriacus, Carica papaya, Cucumis sativus, Eucalyptus grandis, Gossypium raimondii, Kalanchoe fedtschenkoi, Kalanchoe laxiflora, Linum usitatissimum, Musa acuminata, Malus domestica, Mimulus guttatus, Marchantia polymorpha, Oryza sativa, Oropetium thomaeum, Populus trichocarpa, Panicum virgatum, Ricinus communis, Sorghum bicolor, Sphagnum fallax, Solanum lycopersicum, Spirodela polyrhiza, Salix purpurea, and Solanum tuberosum were obtained from the Phytozome database [49]. They all had PPO-KFDV conserved domains. The amino acids of all PPO target sequences were analyzed using ClustalX, and then the phylogenetic tree was constructed using the Neighbor-Joining (NJ) method in MEGA7.0. The accession numbers and gene names are all shown in Table S2.

\section{Analysis of gene and protein structures and motifs}

The Gene Structure Display Server (http://gsds.cbi.pku. edu.cn/) [50] was used to analyze the introns, exons, and UTRs of the 18 poplar PPO genes. The conserved motifs and domains of candidate PPO proteins were identified using MEME (https://meme-suite.org/meme/tools/ meme) [51] and Conserved Domains Database (CDD)
(https://www.ncbi.nlm.nih.gov/Structure/bwrpsb/ bwrpsb.cgi?) [52], respectively. Finally, the conserved motifs and structures of all PPO proteins in poplar were drawn using TBtools (https://github.com/CJ-Chen/ TBtools). Previously described specific parameter settings were used [53].

\section{Chromosomal locations and gene duplication}

The chromosomal positions of PtrPPOs were collected from the Phytozome database [49]. MCScanX was used to analyze PPO gene duplication events [54]. TBtools was used to display the locations and collinearity of PtrPPO genes.

\section{Analysis of cis-regulatory elements}

The promoter sequences (2000 bp upstream of the start codon) of PPOs were analyzed online using Plant-CARE (http://bioinformatics.psb.ugent.be/webtools/plantcare/ $\mathrm{html} /$ ) [55], and all cis-regulatory elements related to hormones and stress were identified.

\section{Transcriptomic data sets to analyze the expression patterns of $P P O$ s}

To evaluate PtrPPOs gene expression profiles, the publicly available transcriptomic data were obtained from PopGenIE (https://popgenie.org/) [56]. In the current study, transcriptome data were collected from stressed plants (drought, mechanical damage, insect beetle damage) and 15 different plant tissues (non-girdled twigs, dormant flowers, expanded flowers, mature leaves, expanding flowers, whole-sucker suckers, mature petiole, prechilling buds, dormant buds, freshly expanded leaves, non-girdled leaves, girdled leaves, mature seeds, young expanding leaves, and dormant cambium phloem) during the growth and development of $P$. trichocarpa. The relative expression of PtrPPOs was displayed as a heat map generated using TBtools [53].

\section{Plant materials and treatments}

Populus (Populus trichocarpa) plant seeds were acquired from Sichuan Agricultural University trail Populus planting base [48]. One-year-old Populus trichocarpa seedlings were planted in a plastic plot $\left(16.0 \mathrm{~h}\right.$ light; $20-25^{\circ} \mathrm{C}$; $70 \%$ air humidity) in Wenjiang, Chengdu, China $\left(30^{\circ} 70^{\prime} \mathrm{N}\right.$, $103^{\circ} 85^{\prime} \mathrm{E}, 537.11 \mathrm{~m}$ above sea level). The plants were watered with $1 \mathrm{~L}$ Hoagland nutrient solution every 2 weeks for 2 months before treatment [46]. Previously, PEG, ABA, and MeJA treatments have been used to investigate the gene responses to abiotic stresses in plants [48, 57, 58]. Sixty-day-old seedlings were treated with PEG, $\mathrm{ABA}$, and MeJA. At least five biological replicates were used for each treatment. For PEG treatments, similarly grown $P$. trichocarpa seedlings $(40-50-\mathrm{cm}$ height, with 30-35 leaves) were treated with 15\% PEG6000 for 0, 1, 3, 
6,9 , and $12 \mathrm{~h}$. For phytohormone analysis, similarly grown $P$. trichocarpa seedlings were treated with a solution containing $200 \mu \mathrm{M}$ ABA (Sigma-Aldrich, Santa Clara, CA, USA) and MeJA (Sigma-Aldrich), for 0, 1, 3, 6, 9, and 12 $h$. The leaves were separated from the plant at the different processing time points, rapidly frozen in liquid nitrogen, and stored in an ultra-low temperature freezer (Thermo Fisher Scientific, Waltham, MA, USA).

Poplar tissues and organs were sampled in accordance with a previously described method [48]. Plant tissues and organs were sampled under stress-free conditions; each plant tissue and organ had at least five biological replicates. We simultaneously collected different $P$. trichocarpa organs and tissues, including young, mature, and old leaves, xylem, phloem, and roots, and immediately immersed them in liquid nitrogen.

\section{RNA extraction and quantitative real-time (qRT-PCR) analysis}

Approximately $1 \mathrm{~g}$ of tissue was isolated for total RNA extraction. Total RNA was prepared using the plant total RNA extraction kit (Aidlab, Beijing, China) according to the manufacturer's protocol, and $2 \mu \mathrm{g}$ of total RNA was reverse transcribed into cDNA using the Tiangen Fast Quant RT Kit (Tiangen Biotech Co. Ltd., Beijing, China). qRT-PCR was conducted as per a previously described protocol [59]. Based on the target gene fragment, Primer Premier 6.0 was used to design primers online; all primers used are listed in Table S1. At least 20 replicates (5 biological replicates $\times 4$ technical replicates) were used per experiment.

\section{Bioinformatic analysis of potential TFs upstream of PtrPPOs}

The plant regulatory network database PlantRegMap (http://plantregmap.gao-lab.org/) [60] was used to identify potential upstream transcriptional factors of PtrPPO9/11/13/14/15. The molecular network regulation map of target genes and upstream transcription factors was drawn using Cytoscape [61]. Transcriptome data for upstream transcription factors under stress conditions (drought and mechanical and insect damage) were obtained from PopGenIE (https://popgenie.org/) [56] and the heat maps were drawn using TBtools.

\section{Availability of data and materials}

The datasets generated and/or analyzed during the current study are available in this article and additional files. The nucleotide and protein sequences of PPOrelated genes in Aquilegia coerulea, Ananas comosus, Amaranthus hypochondriacus, Carica papaya, Cucumis sativus, Eucalyptus grandis, Gossypium raimondii, Kalanchoe fedtschenkoi, Kalanchoe laxiflora, Linum usitatissimum, Musa acuminata, Malus domestica, Mimulus guttatus, Marchantia polymorpha, Oryza sativa, Oropetium thomaeum, Populus trichocarpa, Panicum virgatum, Ricinus communis, Sorghum bicolor, Sphagnum fallax, Solanum lycopersicum, Spirodela polyrhiza, Salix purpurea, and Solanum tuberosum are available in the Phytozome v12.1 database (JGI, https://phytozome.jgi. doe.gov/pz/portal.html).

\section{Statistical analyses}

Microsoft Excel 2020 (Microsoft Corporation, Redmond, WA, USA) and SPSS v.17.0 (SPSS Inc., Chicago, IL, USA) were used to analyze the experimental data. Both one-way analysis of variance was used to determine the significance of the differences among treatments. Student's $t$-test was used to calculate $P$-values ( $P<0.05$; $* P<0.01)$. The data were normalized, and all samples were normally distributed in terms of homogeneity of variance.

\section{Abbreviations}

CDS: Coding sequence; AA: Amino acid; MW: Molecular mass (kDa); GRAVY: Grand average of hydropathicity; PI: Theoretical isoelectric point

\section{Supplementary Information}

The online version contains supplementary material available at https://doi. org/10.1186/s12864-021-08028-9.

\section{Additional file 1.}

\section{Acknowledgments}

Not applicable.

Authors' contributions

FH, YS, QZ, LC, HY, FZ, KZ, XC, JM, JH, and XW conceived and performed the original research project. FH, YS, and QZ performed the experiments. FH, YS, and XW designed the experiments and analyzed the data. FH refined the project and wrote the manuscript with contributions from all authors. FZ and XW supervised the experiments and revised the writing. XW obtained the funding for the research project. All authors read and approved the final manuscript.

\section{Funding}

This work was supported by the National Natural Science Foundation of China (31870645 to XW; 32101481 to FH), and supported by Sichuan Science and Technology Program (2021YFYZ0032 to XW). Furthermore, we appreciate the China Postdoctoral Foundation (2021 M692331 to FH) for supporting this research.

Availability of data and materials

All data supporting the conclusions of this article are provided within the article and its additional files. The genomics sequences data of $A$. thaliana, $O$. sativa, and P. trichocarpa are available in the Phytozome database (https:// phytozome.jgi.doe.gov/pz/portal.html). The public RNA-seq data are available on PopGenIE (https://popgenie.org/gene?id=Potri.001G388900).

\section{Declarations}

Ethics approval and consent to participate

All experiments conducted in this research were in accordance with the IUCN Policy Statement on Research Involving Species at Risk of Extinction and the Convention on the Trade in Endangered Species of Wild Fauna and Flora. The use of $P$. trichocarpa has been licensed. 


\section{Consent for publication}

All the authors of this manuscript give their consent to publish the findings in BMC Genomics.

\section{Competing interests}

The authors declare that there are no conflicts of interest.

\section{Received: 28 February 2021 Accepted: 21 September 2021} Published online: 08 October 2021

\section{References}

1. Yang Y, Li HG, Wang J, Wang HL, He F, Su Y, et al. PeABF3 enhances drought tolerance via promoting $A B A$-induced stomatal closure by directly regulating PeADF5 in poplar. J Exp Bot. 2020;71(22):7270-85. https://doi. org/10.1093/jxb/eraa383.

2. Zhu JK. Abiotic stress signaling and responses in plants. Cell. 2016;167(2): 313-24. https://doi.org/10.1016/j.cell.2016.08.029.

3. Mauch-Mani B, Baccelli I, Luna E, Flors V. Defense priming: An adaptive part of induced resistance. Annu Rev Plant Biol. 2017;68(1):485-512. https://doi. org/10.1146/annurev-arplant-042916-041132.

4. Matsubayashi Y. Posttranslationally modified small-peptide signals in plants. Annu Rev Plant Biol. 2014;65(1):385-413. https://doi.org/10.1146/annurev-a rplant-050312-120122.

5. Zhou JM, Zhang Y. Plant immunity: danger perception and signaling. Cell. 2020;181(5):978-89. https://doi.org/10.1016/j.cell.2020.04.028.

6. Sun Y, Zhu YX, Balint-Kurti PJ, Wang GF. Fine-tuning immunity: players and regulators for plant NLRs. Trends Plant Sci. 2020;25(7):695-713. https://doi. org/10.1016/j.tplants.2020.02.008.

7. Zhao Y, Antoniou-Kourounioti RL, Calder G, Dean C, Howard M. Temperature-dependent growth contributes to long-term cold sensing. Nature. 2020;583(7818):825-9. https://doi.org/10.1038/s41586-020-2485-4

8. Zhao C, Zhang H, Song C, Zhu J-K, Shabala S. Mechanisms of plant responses and adaptation to soil salinity. Innovation. 2020;1(1):100017. https://doi.org/10.1016/j.xinn.2020.100017.

9. Gupta A, Rico-Medina A, Cano-Delgado Al. The physiology of plant responses to drought. Science. 2020;368(6488):266-9. https://doi.org/10.112 6/science.aaz7614

10. Zhang W, Shen Y, Li Z, Xie X, Gong ES, Tian J, et al. Effects of high hydrostatic pressure and thermal processing on anthocyanin content, polyphenol oxidase and beta-glucosidase activities, color, and antioxidant activities of blueberry (Vaccinium Spp.) puree. Food Chem. 2020;342:128564 https://doi.org/10.1016/j.foodchem.2020.128564.

11. Zhang J, Sun X. Recent advances in polyphenol oxidase-mediated plant stress responses. Phytochemistry. 2021;181:112588. https://doi.org/10.1016/j. phytochem.2020.112588.

12. Vamos-Vigyazo L. Polyphenol oxidase and peroxidase in fruits and vegetables. Crit Rev Food Sci Nutr. 1981;15(1):49-127. https://doi.org/10.1 080/10408398109527312.

13. Boeckx T, Winters AL, Webb KJ, Kingston-Smith AH. Polyphenol oxidase in leaves: is there any significance to the chloroplastic localization? J Exp Bot. 2015;66(12):3571-9. https://doi.org/10.1093/jxb/erv141.

14. Cai Y, Dong Z, Zhao S, Han Y, Shao Y, Lu M, et al. Genome-wide analysis of polyphenol oxidase genes and their transcriptional patterns during grain development in Sorghum. Int J Plant Sci. 2013;174(4):710-21. https://doi. org/10.1086/669909.

15. Yu Y, Tang T, Qian Q, Wang Y, Yan M, Zeng D, et al. Independent losses of function in a polyphenol oxidase in rice: differentiation in grain discoloration between subspecies and the role of positive selection under domestication. Plant Cell. 2008;20(11):2946-59. https://doi.org/10.1105/tpc.1 08.060426 .

16. Li D, Deng Z, Liu C, Zhao M, Guo H, Xia Z, et al. Molecular cloning, expression profiles, and characterization of a novel polyphenol oxidase (PPO) gene in Hevea brasiliensis. Biosci Biotechnol Biochem. 2014;78(10): 1648-55. https://doi.org/10.1080/09168451.2014.940828.

17. Stewart RJ, Sawyer BJB, Bucheli CS, Robinson SP. Polyphenol oxidase is induced by chilling and wounding in pineapple. Funct Plant Biol. 2001;28(3): 181-91. https://doi.org/10.1071/PP00094.

18. Zhou Y, O'Hare TJ, Jobin-Decor M, Underhill SJR, Wills RBH, Graham MW. Transcriptional regulation of a pineapple polyphenol oxidase gene and its relationship to blackheart. Plant Biotechnol J. 2003;1(6):463-78. https://doi. org/10.1046/j.1467-7652.2003.00042.x.
19. Gandia-Herrero F, Jimenez M, Cabanes J, Garcia-Carmona F, Escribano J. Tyrosinase inhibitory activity of cucumber compounds: enzymes responsible for browning in cucumber. J Agric Food Chem. 2003;51(26):7764-9. https:// doi.org/10.1021/jf030131u.

20. Wang M, Gao L, Dong S, Sun Y, Shen Q, Guo S. Role of silicon on plantpathogen interactions. Front Plant Sci. 2017:8:701. https://doi.org/10.3389/ fpls.2017.00701.

21. Samynathan R, Perisamy SK, Gandhi S, Anitha J, Sanmugam G, Padmanabhan $\mathrm{M}$, et al. Biochemical and molecular analysis of Camellia sinensis (L.) O. Kuntze tea from the selected P/11/15 clone. J Genet Eng Biotechnol. 2016;14(1):69-75. https://doi.org/10.1016/j.jgeb.2015.12.004.

22. Maioli A, Gianoglio S, Moglia A, Acquadro A, Valentino D, Milani AM, et al. Simultaneous CRISPR/Cas9 editing of three PPO genes reduces fruit flesh Browning in Solanum melongena L. Front Plant Sci. 2020;11:607161. https:// doi.org/10.3389/fpls.2020.607161.

23. Li C, Li D, Li J, Shao F, Lu S. Characterization of the polyphenol oxidase gene family reveals a novel microRNA involved in posttranscriptional regulation of PPOs in Salvia miltiorrhiza. Sci Rep. 2017;7(1):44622. https://doi. org/10.1038/srep44622.

24. Aziz E, Batool R, Akhtar W, Rehman S, Gregersen PL, Mahmood T. Expression analysis of the polyphenol oxidase gene in response to signaling molecules, herbivory and wounding in antisense transgenic tobacco plants. 3 Biotech. 2019;9(2):55.

25. Thipyapong P, Stout MJ, Attajarusit J. Functional analysis of polyphenol oxidases by antisense/sense technology. Molecules. 2007;12(8):1569-95. https://doi.org/10.3390/12081569.

26. Bhonwong A, Stout MJ, Attajarusit J, Tantasawat P. Defensive role of tomato polyphenol oxidases against cotton bollworm (Helicoverpa armigera) and beet armyworm (Spodoptera exigua). J Chem Ecol. 2009;35(1):28-38. https://doi.org/10.1007/s10886-008-9571-7.

27. Wang J, Constabel CP. Polyphenol oxidase overexpression in transgenic Populus enhances resistance to herbivory by forest tent caterpillar (Malacosoma disstria). Planta. 2004;220(1):87-96. https://doi.org/10.1007/ s00425-004-1327-1.

28. Liu D, Meng S, Xiang Z, Yang G, He N. An R1R2R3 MYB Transcription Factor, MnMYB3R1, Regulates the Polyphenol Oxidase Gene in Mulberry (Morus notabilis). Int J Mol Sci. 2019;20(10). https://doi.org/10.3390/ijms20102602.

29. Jansson S, Douglas CJ. Populus: a model system for plant biology. Annu Rev Plant Biol. 2007;58(1):435-58. https://doi.org/10.1146/annurev.arplant.58.032 806.103956

30. Wei M, Xu X, Li C. Identification and expression of CAMTA genes in Populus trichocarpa under biotic and abiotic stress. Sci Rep. 2017;7(1):1-10.

31. Zhuang J, Cai B, Peng RH, Zhu B, Jin XF, Xue Y, et al. Genome-wide analysis of the AP2/ERF gene family in Populus trichocarpa. Biochem Biophys Res Commun. 2008:371(3):468-74. https://doi.org/10.1016/j.bbrc.2008.04.087.

32. Xie T, Chen C, Li C, Liu J, Liu C, He Y. Genome-wide investigation of WRKY gene family in pineapple: evolution and expression profiles during development and stress. BMC Genomics. 2018;19(1):490. https://doi.org/1 0.1186/s12864-018-4880-X.

33. Wang D, Meng S, Su W, Bao Y, Lu Y, Yin W, et al. Genome-Wide Analysis of Multiple Organellar RNA Editing Factor Family in Poplar Reveals Evolution and Roles in Drought Stress. Int J Mol Sci. 2019:20(6):1425.

34. Zhao P, Wang D, Wang R, Kong N, Zhang C, Yang C, et al. Genome-wide analysis of the potato Hsp20 gene family: identification, genomic organization and expression profiles in response to heat stress. BMC Genomics. 2018;19(1):61. https://doi.org/10.1186/s12864-018-4443-1.

35. Zhang L, Zhou D, Hu H, Li W, Hu Y, Xie J, et al. Genome-wide characterization of a SRO gene family involved in response to biotic and abiotic stresses in banana (Musa spp.). BMC Plant Biol. 2019;19(1):211.

36. Moturu TR, Thula S, Singh RK, Nodzynski T, Varekova RS, Friml J, et al. Molecular evolution and diversification of the SMXL gene family. J Exp Bot. 2018;69(9):2367-78. https://doi.org/10.1093/jxb/ery097.

37. Hou J, Ye N, Dong Z, Lu M, Li L, Yin T. Major chromosomal rearrangements distinguish willow and poplar after the ancestral "Salicoid" genome duplication. Genome Biol Evol. 2016;8(6):1868-75. https://doi.org/10.1093/gbe/evw127.

38. Cheng C, Wang Y, Chai F, Li S, Xin H, Liang Z. Genome-wide identification and characterization of the 14-3-3 family in Vitis vinifera $\mathrm{L}$. during berry development and cold- and heat-stress response. BMC Genomics. 2018; 19(1):579.

39. Kaur S, Dhugga KS, Beech R, Singh J. Genome-wide analysis of the cellulose synthase-like (Csl) gene family in bread wheat (Triticum aestivum L.). BMC Plant Biol. 2017;17(1):193. 
40. Thipyapong P, Steffens JC. Tomato polyphenol oxidase (differential response of the polyphenol oxidase F promoter to injuries and wound signals). Plant Physiol. 1997;115(2):409-18. https://doi.org/10.1104/pp.115.2.409.

41. Constabel CP, Yip L, Patton JJ, Christopher ME. Polyphenol oxidase from hybrid poplar. Cloning and expression in response to wounding and herbivory. Plant Physiol. 2000;124(1):285-95. https://doi.org/10.1104/ pp.124.1.285.

42. Chai C, Lin Y, Shen D, Wu Y, Li H, Dou D. Identification and functional characterization of the soybean GmaPPO12 promoter conferring Phytophthora sojae induced expression. PLoS One. 2013;8(6):e67670. https:// doi.org/10.1371/journal.pone.0067670.

43. Zhu H, Chen C, Zeng J, Yun Z, Liu Y, Qu H, et al. MicroRNA528, a hub regulator modulating ROS homeostasis via targeting of a diverse set of genes encoding copper-containing proteins in monocots. New Phytol. 2020;225(1):385-99. https://doi.org/10.1111/nph.16130.

44. Ren G, Wang B, Zhu X, Mu Q, Wang C, Tao R, et al. Cloning, expression, and characterization of miR058 and its target PPO during the development of grapevine berry stone. Gene. 2014;548(2):166-73. https://doi.org/10.1016/j. gene.2014.07.021.

45. Ruiz-Ferrer $V$, Voinnet $O$. Roles of plant small RNAs in biotic stress responses. Annu Rev Plant Biol. 2009;60(1):485-510. https://doi.org/10.1146/annurev.a rplant.043008.092111.

46. He F, Li HG, Wang JJ, Su Y, Wang HL, Feng CH, et al. PeSTZ1, a C2H2-type zinc finger transcription factor from Populus euphratica, enhances freezing tolerance through modulation of ROS scavenging by directly regulating PeAPX2. Plant Biotechnol J. 2019;17(11):2169-83. https://doi.org/10.1111/ pbi.13130.

47. An Y, Zhou Y, Han X, Shen C, Wang S, Liu C, et al. The GATA transcription factor GNC plays an important role in photosynthesis and growth in poplar. J Exp Bot. 2020;71(6):1969-84. https://doi.org/10.1093/jxb/erz564.

48. He F, Shi YJ, Mi JX, Zhao KJ, Cui XL, Chen LH, et al. Genome-Wide Investigation of the NF-X1 Gene Family in Populus trichocarpa Expression Profiles during Development and Stress. Int J Mol Sci. 2021;22(9). https://doi. org/10.3390/ijms22094664.

49. Goodstein DM, Shu S, Howson R, Neupane R, Hayes RD, Fazo J, et al. Phytozome: a comparative platform for green plant genomics. Nucleic Acids Res. 2012;40(Database issue):D1178-86. https://doi.org/10.1093/nar/ gkr944.

50. Guo A, Zhu Q-H, Chen X, Luo J. GSDS: a gene structure display server. Yi chuan. 2007;29(08):1023-6. https://doi.org/10.1360/yc-007-1023.

51. Timothy LB. MEME SUITE: tools for motif discovery and searching. Nucleic Acids Res. 2009:37(Web Server issue):W202-8.

52. Lu S, Wang J, Chitsaz F, Derbyshire M, Geer R, Gonzales N, et al. CDD/ SPARCLE: the conserved domain database in 2020. Nucleic Acids Res. 2019; 48(D1):D265-8. https://doi.org/10.1093/nar/gkz991.

53. Chen $\mathrm{C}$, Chen $\mathrm{H}$, Zhang $Y$, Thomas HR, Frank MH, He Y, et al. TBtools: An integrative toolkit developed for interactive analyses of big biological data Mol Plant. 2020;13(8):1194-202. https://doi.org/10.1016/j.molp.2020.06.009.

54. Wang Y, Tang H, Debarry JD, Tan X, Li J, Wang X, et al. MCScanX: a toolkit for detection and evolutionary analysis of gene synteny and collinearity. Nucleic Acids Res. 2012;40(7):e49. https://doi.org/10.1093/nar/gkr1293.

55. Lescot $M$, Dehais $P$, Thijs $G$, Marchal $K$, Moreau $Y$, Van de Peer $Y$, et al. PlantCARE, a database of plant cis-acting regulatory elements and a portal to tools for in silico analysis of promoter sequences. Nucleic Acids Res. 2002;30(1):325-7. https://doi.org/10.1093/nar/30.1.325.

56. Sundell D, Mannapperuma C, Netotea S, Delhomme N, Lin YC, Sjodin A, et al. The plant genome integrative explorer resource: PlantGenlE.org. New Phytol. 2015;208(4):1149-56. https://doi.org/10.1111/nph.13557.

57. He F, Wang HL, Li HG, Su Y, Li S, Yang Y, et al. PeCHYR1, a ubiquitin E3 ligase from Populus euphratica, enhances drought tolerance via ABAinduced stomatal closure by ROS production in Populus. Plant Biotechnol J. 2018;16(8):1514-28. https://doi.org/10.1111/pbi.12893.

58. Li J, Li Y, Yin Z, Jiang J, Zhang M, Guo X, et al. OsASR5 enhances drought tolerance through a stomatal closure pathway associated with $\mathrm{ABA}$ and $\mathrm{H} 2$ O2 signalling in rice. Plant Biotechnol J. 2017;15(2):183-96. https://doi.org/1 0.1111/pbi.12601.

59. He F, Niu MX, Feng CH, Li HG, Su Y, Su WL, et al. PeSTZ1 confers salt stress tolerance by scavenging the accumulation of ROS through regulating the expression of PeZAT12 and PeAPX2 in Populus. Tree Physiol. 2020;40(9): 1292-311. https://doi.org/10.1093/treephys/tpaa050.
60. Tian F, Yang DC, Meng YQ, Jin J, Gao G. PlantRegMap: charting functional regulatory maps in plants. Nucleic Acids Res. 2020;48(D1):D1104-13. https:// doi.org/10.1093/nar/gkz1020.

61. Shannon P, Markiel A, Ozier O, Baliga NS, Wang JT, Ramage D, et al. Cytoscape: a software environment for integrated models of biomolecular interaction networks. Genome Res. 2003;13(11):2498-504. https://doi.org/1 $0.1101 /$ gr.1239303.

\section{Publisher's Note}

Springer Nature remains neutral with regard to jurisdictional claims in published maps and institutional affiliations.
Ready to submit your research? Choose BMC and benefit from:

- fast, convenient online submission

- thorough peer review by experienced researchers in your field

- rapid publication on acceptance

- support for research data, including large and complex data types

- gold Open Access which fosters wider collaboration and increased citations

- maximum visibility for your research: over $100 \mathrm{M}$ website views per year

At BMC, research is always in progress.

Learn more biomedcentral.com/submissions 